\title{
Patient-Reported Satisfaction after Prophylactic Operations of the Breast
}

\author{
Katja Keller ${ }^{a, b, c} \quad$ Cornelia Meisel $^{a, b, c}$ Nannette Grübling ${ }^{a, b, c}$ \\ Andrea Petzold ${ }^{a, b, c}$ Pauline Wimberger ${ }^{a, b, c}$ Karin Kast ${ }^{a, b, c}$ \\ ${ }^{a}$ Department of Gynecology and Obstetrics, Medical Faculty and University Hospital Carl Gustav Carus, Technische \\ Universität Dresden, Dresden, ${ }^{\mathrm{b}}$ National Center for Tumor Diseases (NCT), Partner Site Dresden, Dresden, and \\ ${ }^{c}$ German Cancer Consortium (DKTK), Dresden and German Cancer Research Center (DKFZ), Heidelberg, Germany
}

\section{Keywords}

Prophylactic mastectomy · Satisfaction $\cdot$ BRCA1/BRCA2

mutations · Long-term follow-up · Breast cancer

\begin{abstract}
Background: Prophylactic mastectomies in carriers of mutations in BRCA1 or BRCA2 are becoming increasingly more accepted. We investigated the outcome after prophylactic mastectomy, especially regarding satisfaction with the procedure, in a monocenter study. Methods: BRCA1/2 mutation carriers and non-carriers with elevated pedigree-based cancer risk were followed prospectively in a structured surveillance program between 2000 and 2017. A retrospective telephone survey was conducted among all patients with documented prophylactic mastectomy. Complications and satisfaction with the decision for prophylactic mastectomy were recorded. Results: 39 patients who opted for a prophylactic mastectomy (38 BRCA1/2 mutation carriers and 1 noncarrier) were interviewed. Mostly nipple-sparing mastectomy with reconstruction was performed (87\%). Half of the patients $(22 / 39 ; 56.4 \%)$ had a history of unilateral breast cancer. The median time since prophylactic mastectomy was 5.6 years. While $61.5 \%$ did not report any complications, flap loss was seen in 15\% (3/20) and moderate limitations in everyday life were present in 20\% (7/35). An improvement in quality of life was noticed by $82 \%$ after prophylactic mastectomy and no patient expressed regret with regard to the decision. Conclusions: Prophylactic mastectomy is a procedure with
\end{abstract}

\section{KARGER}

๑) 2019 S. Karger AG, Basel risk for long-term complications in some cases. Our results confirm high satisfaction with the decision and improved quality of life.

() 2019 S. Karger AG, Basel

\section{Introduction}

$B R C A 1 / 2$ mutation carriers face a high lifelong risk for breast and ovarian cancer. For both genes, the breast cancer risk until the age of 80 years is $70 \%$, and for ovarian cancer it is $40 \%$ and $17 \%$ for $B R C A 1$ and $B R C A 2$, respectively [1]. Intensified screening programs with breast magnetic resonance imaging (MRI) aim to improve the prognosis of breast cancers through early detection, and there is evidence for a survival benefit by surveillance [2]. But mutation carriers increasingly opt for risk-reducing operations of the breast. For healthy carriers, not only a reduction of $90-95 \%$ in the risk of subsequent breast cancer was shown by bilateral prophylactic mastectomy, but also improved overall survival was described [3]. In patients with unilateral breast cancer, the benefit of contralateral prophylactic mastectomy is less clear $[4,5]$. Prior to the risk-reducing surgery, comprehensive counseling at different time points is necessary. Counseling should take place in specialized centers and should contain different reconstruction methods, such as the use of implants and autologous tissue. The decision is guided by a woman's activity and plans, body shape, and personal preferences. 
Table 1. Questionnaire for the telephone interview

\begin{tabular}{|c|c|c|c|c|c|}
\hline \multirow{2}{*}{$\begin{array}{l}\text { Questions } \\
\text { Type of mastectomy }\end{array}$} & \multicolumn{5}{|c|}{ Spectrum of answers } \\
\hline & MRM & SM & & & \\
\hline Type of reconstruction & none & implant & autologous tissue & & \\
\hline Number of operations & total & & & & \\
\hline Complications after surgery & none & moderate & severe & & \\
\hline Satisfaction with cosmetic result & not at all & rather unsatisfied & indifferent & satisfied & very satisfied \\
\hline Pain & none & little & moderate & strong & severe \\
\hline Improvement of quality of life & none & little & moderate & severe & \\
\hline Limitations in everyday life & none & little & moderate & severe & \\
\hline Limitations in leisure time and activities & none & little & moderate & severe & \\
\hline Same decision for prophylactic surgery again? & yes & no & different & & \\
\hline Negative impact on family/partnership? & yes & no & indifferent & & \\
\hline Salpingo-oophorectomy & yes & no & & & \\
\hline
\end{tabular}

MRM, modified radical mastectomy; SM, subcutaneous mastectomy.

Satisfaction of patients depends on the esthetic outcome, freedom from lasting symptoms with limitations in daily life, and reduction of anxiety after surgery. According to 1 meta-analysis, most patients would decide to have the same procedure performed again [5]. But after unilateral sporadic breast cancer, reduced satisfaction of patients with contralateral prophylactic mastectomy with one or more unplanned following surgeries was described [6]. In addition, more body image problems and less sexual pleasure, but less anxiety and improved social activities were reported in a study of women with familial risk for breast cancer at 1 year after prophylactic bilateral breast cancer [7].

With this study, we aimed to investigate the complication rate and satisfaction of carriers at the Dresden center of the German Consortium Hereditary Breast and Ovarian Cancer (GC-HBOC) after prophylactic operations of the breast with and without prior breast cancer diagnosis.

\section{Methods}

Between 2000 and 08/2017, all patients who fulfilled certain criteria of familial cancer risk were counseled interdisciplinarily, and molecular genetic analysis was carried out as described previously at the Dresden center of the GC-HBOC [8]. Prophylactic risk-reducing operations were offered to all women with a pathologic mutation in BRCA1 or BRCA2. After consenting to participate in the study, each family was documented in the mutual database of the GC-HBOC. Follow-up was performed for all women who took part in our center's intensified surveillance program or by personal contact with carriers and individuals with very high pedigree-based risk who sought additional counseling for prophylactic operations. All women after risk-reducing bilateral mastectomy are offered to undergo one last MRI to rule out remaining breast tissue in order to objectify the surgical outcome under research conditions 1 year after the procedure.

Between 04/2017 and 08/2017, a telephone survey was conducted among all patients with a documented history of prophy- lactic surgery of the breast in our database. Altogether, 44 women were eligible, of whom 39 were interviewed by a breast surgeon and 5 were lost to follow-up. The interview consisted of 18 questions on type of surgery, status of reconstruction, early and late complications, and number of operations (table 1). Moreover, satisfaction, limitations in daily life, and extent of anxiety reduction were enquired about (table 1).

The data cut of the database was 08/2017. Statistical analyses were performed with SPSS.

\section{Results}

Altogether, 1,319 families were counseled at the Dresden center, with 330 families carrying a pathogenic mutation in BRCA1 or BRCA2, of which roughly $11 \%$ of the women at risk opted for a prophylactic mastectomy. Of the 39 patients with documented prophylactic mastectomy and a telephone interview, 38 were carriers of a pathogenic mutation. 1 patient with prophylactic mastectomy without $B R C A 1 / 2$ mutation was from a family with very high pedigree-based risks. The median age at the time of the interview was 47 years. The median time since breast cancer in affected individuals was 7.8 years and the median time since prophylactic mastectomy was 5.6 years. The characteristics of the participants of the telephone interview are displayed in table 2.

In $43.6 \%$ (17/39) of the women, an exclusively prophylactic operation was performed (bilateral mastectomy in a healthy individual). About half of the patients (22/39; $56.4 \%$ ) were affected with unilateral breast cancer (table 3), some of whom had a primary mastectomy, e.g. after neoadjuvant chemotherapy, while others opted for a secondary bilateral mastectomy after initial breast-conserving surgery (table 2). Of the 39 patients under investigation, $25(64.1 \%)$ reported having undertaken a bilateral prophylactic salpingo-oophorectomy (table 2). 
Table 2. Characteristics of the participants in the telephone interview

\begin{tabular}{lrc}
\hline Characteristics & $\mathrm{n}$ & Value \\
\hline Participants, all & 39 & \\
$\quad$ Healthy mutation carrier & 17 & \\
$\quad$ Healthy without BRCA1/2 mutation & 1 & \\
$\quad$ Patients with unilateral breast cancer & 21 & \\
Age at time of interview, years, median (range) & 39 & $47(26-75)$ \\
Time since breast cancer, years, median (range) & 21 & $7.8(3-33)$ \\
Time since prophylactic mastectomy, years, median (range) & 39 & $5.6(1-32)$ \\
Prophylactic salpingo-oophorectomy, yes/no & 39 & $24 / 15$ \\
Prior chemotherapy, yes/no & 21 & $17 / 4$ \\
Prior radiation therapy, yes/no & 21 & $5 / 16$ \\
\hline
\end{tabular}

Most of the patients with bilateral mastectomy were treated with nipple-sparing subcutaneous mastectomy $(34 / 39 ; 85 \%)$ and alloplastic or autologous reconstruction, while some wished to receive a modified bilateral mastectomy (10\%) without reconstruction (fig. 1a). All of the latter were unilaterally affected carriers of mutations in BRCA1 or BRCA2.

Of the 35 patients with reconstruction, 43\% (15/35) opted for breast implants, while 57\% (20/35) were reconstructed with autologous tissue (fig. 1b). Reconstruction with implants was mostly performed during a single surgery $(12 / 15 ; 80 \%) .2$ patients needed 2 different surgeries and 1 patient had her implants successfully placed only after 4 surgeries, due to wound healing problems. For autologous reconstruction, a median of 3 surgeries were necessary. Only 4 out of 20 patients (20\%) with autologous reconstruction had their surgery completed within a single operation.

Reconstruction was performed without any complications in $61.5 \%$ (24/39) of the patients and moderate complication such as infection or rebleeding occurred in 11 patients $(11 / 39 ; 28.2 \%) .3$ women $(7.7 \%)$ faced severe complications: 2 with loss of the transplanted tissue flap and 1 with loss of the breast implant (fig. 1d). No lifethreatening complications were observed.

Most patients were satisfied or very satisfied with the cosmetic outcome of their prophylactic mastectomy at the time of the interview $(33 / 39 ; 85 \%)$. Only 4 reported discontent $(4 / 39 ; 10 \%)$ with the cosmetic outcome and 2 $(2 / 39 ; 5.1 \%)$ would elect a different type of operation. For example, one of these patients would prefer a subcutaneous rather than a subpectoral location for her implant. Patients with autologous reconstruction displayed higher satisfaction with the result $(19 / 20 ; 95 \%)$ compared to those operated with implant reconstruction (11/15; 73.3\%). Moreover, patients after unilateral breast cancer were more often satisfied with the result $(16 / 18 ; 88.9 \%)$ than healthy carriers $(14 / 17 ; 82.4 \%)$.

In terms of long-term surgery-associated morbidity, $59 \%(23 / 39)$ of the patients had no pain. Severe pain was not reported by any of the patients. About half of the pa-
Table 3. Tumor characteristics

$\mathrm{n}$

\begin{tabular}{lr}
\hline Size of tumor & \\
Tis & 1 \\
T1 & 13 \\
T2 & 6 \\
T3/T4 & 0 \\
Unknown & 2 \\
\hline Total & 22 \\
\hline
\end{tabular}

\begin{tabular}{lr}
\hline Nodal status & 13 \\
N0 & 6 \\
N1 & 2 \\
Unknown & 21 \\
\hline Total & 22 \\
\hline Metastases & 0 \\
M0 & 0 \\
M1 & 22 \\
Unknown
\end{tabular}

Tumor biology

HR+, HER2- 6

HR-, HER2- 12

HR-, HER2+ 1

HR+, HER $2+$

Unknown 2

Total 22

HR, hormone receptor; HER2, human epidermal growth factor receptor 2 .

tients experienced no limitations in everyday life (20/39; $51.3 \%$ ) or in the selection of their recreational activities or hobbies $(25 / 39 ; 64.1 \%)$. Severe limitations were not reported by any patient, moderate limitations in everyday life were reported by 7 patients $(7 / 39 ; 18 \%)$, and moderate limitations in leisure time activities by $4(4 / 39 ; 10.3 \%)$ (fig. 1e). A moderate restriction in everyday life was, for example, that it was no longer possible to lift or carry 


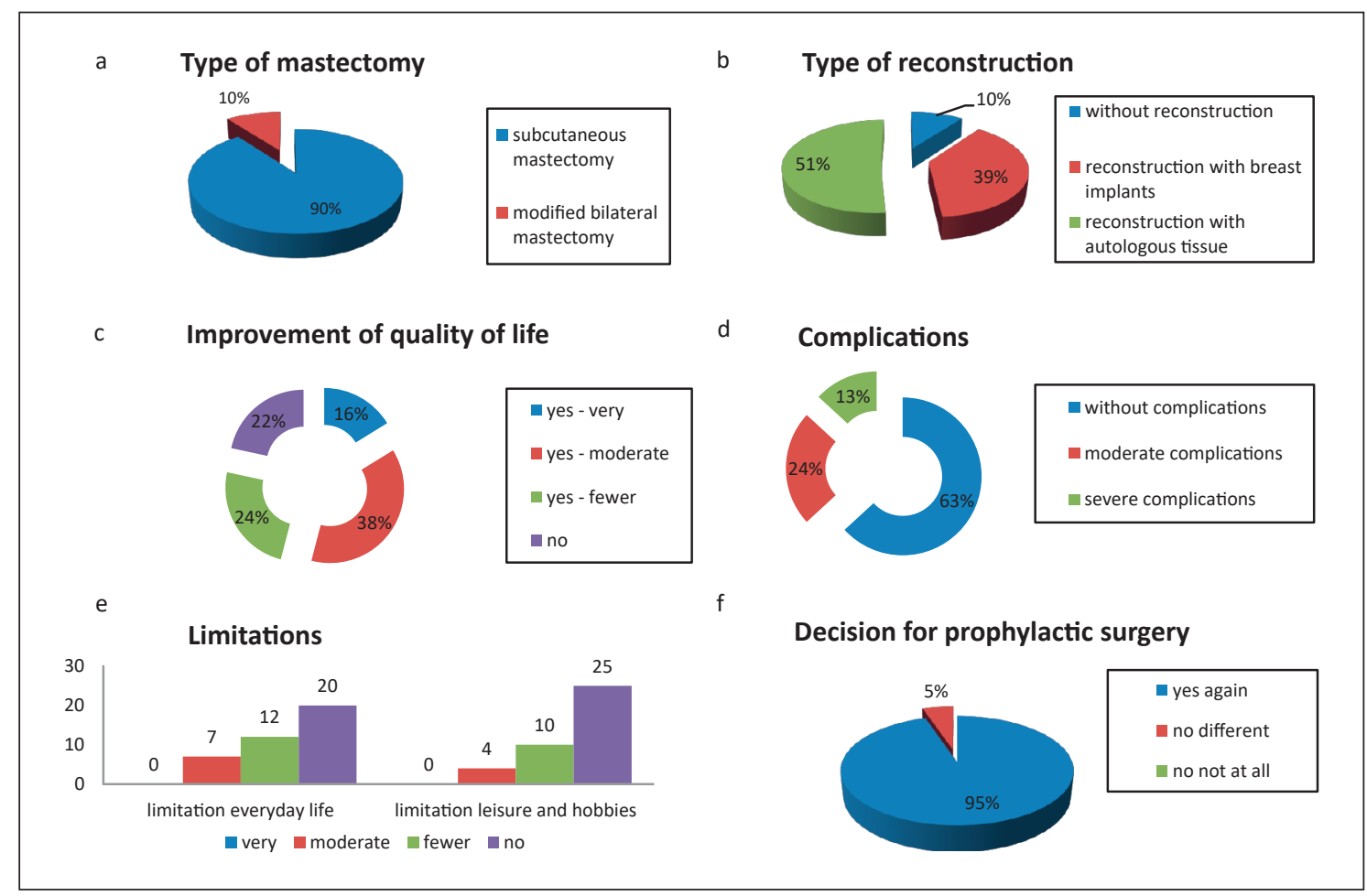

Fig. 1. Results of the telephone interview. a Type of mastectomy, $\mathbf{b}$ type of reconstruction, $\mathbf{c}$ improvement of quality of life, $\mathbf{d}$ Complications, $\mathbf{e}$ Limitation in everyday life/leisure time activities, $\mathbf{f}$ decision for prophylactic surgery.

heavy weight, and in leisure time activities that a prone position was no longer possible.

Improvement in quality of life was seen in $78 \%$ of patients, with reduction of anxiety being the most important reason (fig. 1c). While $95 \%$ of the patients would opt for the same procedure again, 5.1\% would choose another type of surgery. None of the 39 individuals under investigation reported regrets in terms of their decision for prophylactic mastectomy (fig. 1f). One of the patients had a local recurrence, but none developed metastatic disease.

\section{Discussion}

We searched our database for women with documented prophylactic mastectomy after interdisciplinary counseling and molecular genetic analysis of the breast cancer genes in our GC-HBOC center in Dresden. With about $11 \%$, the percentage of carriers who decided to undergo prophylactic mastectomy was low. About half of them had a history of breast cancer, although overall survival benefit has primarily been shown in healthy carriers. In the past, differences in uptake of prophylactic mastectomy were reported among different cultures. Compared to more conservative countries such as France and Poland, uptake in countries such as the USA, Sweden or The
Netherlands was higher [9-12]. With the outing of Angelina Jolie in 2013, the procedure lost its taboo, and we noticed an increase in requests for the surgery at our center. Only recently did another center of the GC-HBOC report a higher rate of prophylactic mastectomy, namely $27 \%$ and $44 \%$ among affected and healthy BRCA $1 / 2 \mathrm{mu}-$ tation carriers, respectively [13]. This difference might be mainly explained by the different approach they adopted in their study. Schott et al. [13] sent questionnaires to all patients who were counseled at the center in Heidelberg, whereas we recorded operations that were actively communicated. However, cultural reasons cannot be completely excluded in comparing centers in the former western part of Germany with those in the former eastern part.

Unplanned additional operations, wound healing problems, and flap loss were described as negative side effects in the meta-analysis of Lostumbo et al. [5]. In a recent one-center study, the overall complication rate was reported to lie at $15.9 \%$, with significantly less complications after autologous reconstruction compared to alloplastic reconstruction [14]. Generally, wound infections and seromas are more common in alloplastic breast implants [14, 15]. Autologous reconstruction was reported to be associated with longer hospital stays, but also with fewer operations, less reconstruction failures 
(7.3 vs. $1.3 \%)$ and a shorter time to full achievement of reconstruction [16-18]. Complication rates are higher after neoadjuvant chemotherapy or after radiation therapy in smoking or obese patients [19]. After alloplastic breast reconstruction, Mousa et al. [20] reported postoperative complications in $45 \%$ of patients, of which $29 \%$ occurred after prior neoadjuvant chemotherapy and $25 \%$ after prior radiotherapy. In $30 \%$ of patients, revisional surgery (closure of wound, debridement, exchange or removal of implant) was needed. In our cohort, the overall complication rate was $38 \%$, which might be due to the high percentage of women participating in the study after treatment for prior breast cancer. With the exception of 2 women, all patients would elect the same procedure again. The number of severe complications such as flap loss after autologous reconstruction is rather high. The operations took place in different hospitals all over Germany. In order to guarantee high oncologic safety and the best possible aesthetic outcome with low complication rates, these complex operations should only be performed in centers with interdisciplinary teams of oncologic and plastic surgeons $[21,22]$. But the patient's wish is not regulated further, for instance by limited insurance coverage.

In spite of complications, most women after prophylactic mastectomy reported high satisfaction with their decision in the meta-analysis of Lostumbo et al. [5]. This is in accordance with our findings. Even in cases of dissatisfaction with the cosmetic outcome, all our patients would opt to undergo the risk-reducing operation again.

Satisfaction with cosmetic outcome was higher after autologous reconstruction, which is in accordance with the literature [17, 23-27]. But due to the small numbers in our analysis and differences in preconditions for the individual woman, this implies no general advice to prefer one method over the other. In our study, patients after unilateral breast cancer reported higher satisfaction compared to healthy patients. Although this was expected, one other group found partly different results. Kazzazi et al. [24] describe higher satisfaction among healthy individuals or after bilateral breast cancer with mastectomy and reconstruction compared to those with unilateral breast cancer with bilateral mastectomy and reconstruction. As an explanation, lack of time for making the decision for contralateral mastectomy is suggested.

Most study participants reported improvement in quality of life primarily due to reduction of anxiety. Those with unchanged quality of life reported having taken a rational decision without feeling stressed by anxiety before the operation. With a mean follow-up time of 14.5 years, a retrospective study by Frost et al. [28] found satisfaction with the procedure in $70 \%$ of all patients. More- over, increase in emotional stability and decrease in stress was perceived in about $25 \%$ of patients. A number of smaller studies with a short follow-up of 1-2 years provide similar data $[7,28-31]$, but long-term prospective studies are still needed for the comprehensive counseling of patients at high risk for breast cancer.

The strength of our analysis lies in the qualified telephone interview conducted by a breast surgeon. Especially for patients with prior unilateral breast cancer, it is sometimes challenging to answer questions on intention, number of surgeries, or type of complication. A limitation of our study is the restricted follow-up of the 1,319 families at our center. Not all families are represented with at least 1 participant in our intensified surveillance program. Therefore, underestimation of the rate of uptake of prophylactic mastectomies is possible. Moreover, our sample is too small to compare the impact of type of mastectomy and reconstruction on complications and quality of life. It should also be mentioned that no validated questionnaire was used to enquire about patient satisfaction.

Prophylactic mastectomy is the most effective procedure to improve survival rates in healthy carriers of a mutation in BRCA1/2 and possibly also in unilaterally affected carriers. As we can confirm, psychosocial benefits outweigh possible early and late side effects of the surgery. Knowledge on genetic and non-genetic risk modifiers might help to define individual risk and the timing of the procedure in the future.

\section{Acknowledgements}

We thank Rita Schmutzler, Christoph Engel, and all investigators of the GC-HBOC for establishing the background on the basis of which this work was possible. At the center in Dresden, we especially thank Dominique Weise, Sylke Schmidtke, Kristin Eichhorn, and Nannette Kranz for their continuous commitment in patient management, documentation, and analysis. We thank Evelin Schröck, Andreas Rump, Karl Hackmann, Arne Jahn, and Johannes Wagner for genetic counseling and analysis. We thank all the patients for their individual contribution to this research. Finally, we thank the German Cancer Aid for their support of the GC-HBOC with grant no. 110837.

\section{Statement of Ethics}

The study protocol has been approved by the research institute's committee on human research.

\section{Funding Sources}

German Cancer Aid grant no. 110837 for GC-HBOC. 


\section{Author Contributions}

K.Ke.: Conception of the work, analysis and interpretation of data, writing of the manuscript, final approval of the version to be published; C.M.: conception of the work, acquisition, interpretation of the data, final approval of the version to be published; K. Ka.: conception of the work, acquisition, interpretation of data, critical revision of the manuscript for important intellectual content, final approval of the version to be published; all other authors: acquisition of data, critical revision of the manuscript for important intellectual content, final approval of publication.

\section{Disclosure Statement}

The authors have no conflicts of interest to declare.

\section{References}

${ }_{1}$ Kuchenbaecker KB, Hopper JL, Barnes DR, et al: Risks of breast, ovarian, and contralateral breast cancer for BRCA1 and BRCA2 mutation carriers. JAMA 2017;317:2402-2416.

-2 Evans DG, Harkness EF, Howell A, Wilson M, Hurley E, Holmen MM, Tharmaratnam KU, Hagen AI, Lim Y, Maxwell AJ, Moller P: Intensive breast screening in BRCA2 mutation carriers is associated with reduced breast cancer specific and all cause mortality. Hered Cancer Clin Pract 2016;14:8.

3 Hartmann LC, Lindor NM: The role of riskreducing surgery in hereditary breast and ovarian cancer. N Engl J Med 2016;374:454468.

-4 Heemskerk-Gerritsen BA, Rookus MA, Aalfs CM, Ausems MG, Collee JM, Jansen L, Kets CM, Keymeulen KB, Koppert LB, MeijersHeijboer HE, Mooij TM, Tollenaar RA, Vasen $\mathrm{HF}$, Hooning MJ, Seynaeve C: Improved overall survival after contralateral risk-reducing mastectomy in BRCA1/2 mutation carriers with a history of unilateral breast cancer: a prospective analysis. Int J Cancer 2015;136 668-677.

5 Lostumbo L, Carbine N, Wallace J, Ezzo J: Prophylactic mastectomy for the prevention of breast cancer. Cochrane Database Syst Rev 2004; (4):CD002748.

6 Boughey JC, Hoskin TL, Hartmann LC, Johnson JL, Jacobson SR, Degnim AC, Frost MH: Impact of reconstruction and reoperation on long-term patient-reported satisfaction after contralateral prophylactic mastectomy. Ann Surg Oncol 2015;22:401-408.

-7 Brandberg Y, Sandelin K, Erikson S, Jurell G, Liljegren A, Lindblom A, Linden A, von Wachenfeldt A, Wickman M, Arver B: Psychological reactions, quality of life, and body image after bilateral prophylactic mastectomy in women at high risk for breast cancer: a prospective 1-year follow-up study. J Clin Oncol 2008;26:3943-3949.

-8 Meisel C, Sadowski CE, Kohlstedt D, Keller K, Staritz F, Grubling N, Becker K, Mackenroth L, Rump A, Schrock E, Arnold N, Wimberger $\mathrm{P}$, Kast K: Spectrum of genetic variants of BRCA1 and BRCA2 in a German single center study. Arch Gynecol Obstet 2017;295:12271238 .
-9 Arver B, Isaksson K, Atterhem H, Baan A, Bergkvist L, Brandberg Y, Ehrencrona $\mathrm{H}$, Emanuelsson M, Hellborg H, Henriksson K, Karlsson P, Loman N, Lundberg J, Ringberg A, Askmalm MS, Wickman M, Sandelin K: Bilateral prophylactic mastectomy in Swedish women at high risk of breast cancer: a national survey. Ann Surg 2011;253:1147-1154.

10 Gronwald J, Byrski T, Huzarski T, Cybulski C, Stawicka M, Szwiec M, Debniak T, Tulman A, Sun P, Oszurek O, Lubinski J, Narod SA: A survey of preventive measures among $B R C A 1$ mutation carriers from Poland. Clin Genet 2007;71:153-157.

$>11$ Julian-Reynier C, Bouhnik AD, MouretFourme E, Gauthier-Villars M, Berthet P, Lasset C, Fricker JP, Caron O, Gesta P, Luporsi E, Faivre L, Longy M, Gladieff L, Frenay M, Dreyfus H, Sobol H, Vennin P, Nogues C: Time to prophylactic surgery in BRCA $1 / 2$ carriers depends on psychological and other characteristics. Genet Med 2010;12:801-807.

12 Meijers-Heijboer H, Brekelmans CT, MenkePluymers M, Seynaeve C, Baalbergen A, Burger C, Crepin E, van den Ouweland AW, van Geel B, Klijn JG: Use of genetic testing and prophylactic mastectomy and oophorectomy in women with breast or ovarian cancer from families with a $B R C A 1$ or $B R C A 2$ mutation. J Clin Oncol 2003;21:1675-1681.

13 Schott S, Vetter L, Keller M, Bruckner T, Golatta M, Eismann S, Dikow N, Evers C, Sohn C, Heil J: Women at familial risk of breast cancer electing for prophylactic mastectomy: frequencies, procedures, and decision-making characteristics. Arch Gynecol Obstet 2017; 295:1451-1458.

14 Bletsis P, Bucknor A, Chattha A, Kamali P, Chen A, Flecha-Hirsch R, van der Lei B, Lee BT, Lin SJ: Evaluation of contralateral and bilateral prophylactic mastectomy and reconstruction outcomes: comparing alloplastic and autologous reconstruction. Ann Plast Surg 2018;80(suppl 4):S144-S149.

15 Dassoulas KR, Wang J, Thuman J, Ndem I, Schaeffer C, Stovall M, Tilt A, Lee A, Lin KY, Campbell CA: Reducing infection rates in implant-based breast reconstruction: impact of an evidence-based protocol. Ann Plast Surg 2018;80:493-499.
16 Fischer JP, Nelson JA, Cleveland E, Sieber B, Rohrbach JI, Serletti JM, Kanchwala S: Breast reconstruction modality outcome study: a comparison of expander/implants and free flaps in select patients. Plast Reconstr Surg 2013;131:928-934.

17 Atisha DM, Rushing CN, Samsa GP, Locklear TD, Cox CE, Shelley Hwang E, Zenn MR, Pusic AL, Abernethy AP: A national snapshot of satisfaction with breast cancer procedures. Ann Surg Oncol 2015;22:361-369.

18 Alderman AK, Kuhn LE, Lowery JC, Wilkins EG: Does patient satisfaction with breast reconstruction change over time? Two-year results of the Michigan Breast Reconstruction Outcomes Study. J Am Coll Surg 2007;204: 7-12.

19 Chang EI, Chang EI, Soto-Miranda MA, Zhang H, Nosrati N, Ghali S, Chang DW: Evolution of bilateral free flap breast reconstruction over 10 years: optimizing outcomes and comparison to unilateral reconstruction. Plast Reconstr Surg 2015;135:946e-953e.

20 Mousa M, Barnea Y, Arad U, Inbal A, Klausner J, Menes T: Association between postoperative complications after immediate alloplastic breast reconstruction and oncologic outcome. Clin Breast Cancer 2018;18:e699e702.

21 Chang EI, Chang EI, Soto-Miranda MA, Zhang H, Nosrati N, Crosby MA, Reece GP, Robb GL, Chang DW: Comprehensive evaluation of risk factors and management of impending flap loss in 2138 breast free flaps. Ann Plast Surg 2016;77:67-71.

22 Tuggle CT, Patel A, Broer N, Persing JA, Sosa JA, Au AF: Increased hospital volume is associated with improved outcomes following abdominal-based breast reconstruction. J Plastic Surg Hand Surg 2014;48:382-388.

23 Jeevan R, Browne JP, Gulliver-Clarke C, Pereira J, Caddy CM, van der Meulen JH, Cromwell DA: Association between age and access to immediate breast reconstruction in women undergoing mastectomy for breast cancer. Br J Surg 2017;104:555-561.

24 Kazzazi F, Haggie R, Forouhi P, Kazzazi N, Wyld L, Malata CM: A comparison of patient satisfaction (using the BREAST-Q questionnaire) with bilateral breast reconstruction following risk-reducing or therapeutic mastectomy. J Plast Reconstr Aesthet Surg 2018;71: 1324-1331. 
25 Moberg IO, Schou Bredal I, Schneider MR, Tonseth KA, Schlichting E: Complications, risk factors, and patients-reported outcomes after skin-sparing mastectomy followed by breast reconstruction in women with $B R C A$ mutations. J Plast Surg Hand Surg 2018;52: 234-239.

26 Santosa KB, Qi J, Kim HM, Hamill JB, Wilkins EG, Pusic AL: Long-term patient-reported outcomes in postmastectomy breast reconstruction. JAMA Surg 2018;153:891-899.

-27 van Verschuer VMT, Mureau MAM, Heemskerk-Gerritsen BAM, Gadjradj PS, Rogier C, Verhoef C, Gopie JP, Seynaeve C, Koppert LB: Long-term outcomes of bilateral direct-toimplant breast reconstruction in women at high breast cancer risk. J Plast Surg Hand Surg 2018;52:245-252.
8 Frost MH, Schaid DJ, Sellers TA, Slezak JM, Arnold PG, Woods JE, Petty PM, Johnson JL, Sitta DL, McDonnell SK, Rummans TA, Jenkins RB, Sloan JA, Hartmann LC: Long-term satisfaction and psychological and social function following bilateral prophylactic mastectomy. JAMA 2000;284:319-324.

9 Hatcher MB, Fallowfield L, A'Hern R: The psychosocial impact of bilateral prophylactic mastectomy: prospective study using questionnaires and semistructured interviews. BMJ 2001;322:76.
0 Heiniger L, Butow PN, Coll J, Bullen T, Wilson J, Baylock B, Meiser B, Price MA: Longterm outcomes of risk-reducing surgery in unaffected women at increased familial risk of breast and/or ovarian cancer. Fam Cancer 2015;14:105-115.

-31 Unukovych D, Sandelin K, Liljegren A, Arver B, Wickman M, Johansson $\mathrm{H}$, Brandberg Y: Contralateral prophylactic mastectomy in breast cancer patients with a family history: a prospective 2-years follow-up study of health related quality of life, sexuality and body image. Eur J Cancer 2012;48:3150-3156. 